\title{
Cat amniotic membrane multipotent cells are nontumorigenic and are safe for use in cell transplantation
}

This article was published in the following Dove Press journal:

Stem Cells and Cloning:Advances and Applications

27 August 2014

Number of times this article has been viewed

\author{
Atanasio S Vidane' \\ Aline F Souza' \\ Rafael V Sampaio' \\ Fabiana F Bressan ${ }^{2}$ \\ Naira C Pieri' \\ Daniele S Martins ${ }^{2}$ \\ Flavio V Meirelles ${ }^{2}$ \\ Maria A Miglino' \\ Carlos E Ambrósio² \\ 'Department of Surgery, Faculty of \\ Veterinary Medicine and Animal \\ Science, University of São Paulo, \\ São Paulo, Brazil; ${ }^{2}$ Department of \\ Veterinary Medicine, Faculty of \\ Animal Sciences and Food \\ Engineering, University of \\ São Paulo, São Paulo, Brazil
}

\begin{abstract}
Amnion-derived mesenchymal stem cells (AMSCs) are multipotent cells with an enhanced ability to differentiate into multiple lineages. AMSCs can be acquired through noninvasive methods, and therefore are exempt from the typical ethical issues surrounding stem cell use. The objective of this study was to isolate and characterize AMSCs from a cat amniotic membrane for future application in regenerative medicine. The cat AMSCs were harvested after mechanical and enzymatic digestion of amnion. In culture medium, the cat AMSCs adhered to a plastic culture dish and displayed a fibroblast-like morphology. Immunophenotyping assays were positive for the mesenchymal stem cell-specific markers CD73 and CD90 but not the hematopoietic markers CD34, CD45, and CD79. Under appropriate conditions, the cat AMSCs differentiated into osteogenic, chondrogenic, and adipogenic cell lineages. One advantage of cat AMSCs was nonteratogenicity, assessed 4 weeks post injection of undifferentiated AMSCs into immunodeficient mice. These findings suggest that cat amniotic membranes may be an important and useful source of mesenchymal stem cells for clinical applications, especially for cell or tissue replacement in chronic and degenerative diseases.
\end{abstract}

Keywords: amnion, cats, cell differentiation, fetal membranes, mesenchymal cells

\section{Introduction}

Mesenchymal stem cells (MSCs) have awakened interest in regenerative medicine due to their high capability to proliferate and differentiate into specialized lineages under specific stimuli. ${ }^{1,2}$ MSCs are multipotent adult somatic stem cells derived from bone marrow and also from a wide variety of organs, including the reproductive system, eg, the endometrium and ovaries, and also postnatal organs. ${ }^{3-5}$ Extraembryonic and fetal tissue-derived stem cells have recently received increased interest in the field of regenerative medicine. ${ }^{6-10}$ These cells have an enhanced ability for differentiation into multiple specialized lineages under specific conditions, can be acquired through noninvasive methods and are exempt from ethical issues because the tissues they come from are discarded after birth.,11-14 The amniotic membrane is widely used in tissue engineering to promote wound epithelialization ${ }^{15}$ and ocular surface reconstruction. ${ }^{16}$

The amniotic membrane comprises two types of cells, ie, amniotic epithelial stem cells that line the inner surface of the amnion and amniotic mesenchymal stem cells (AMSCs) that are dispersed throughout the stroma. Given their immunomodulatory properties, anti-inflammatory functions, and nontumorigenicity, AMSCs are a promising tool in cell therapy and regenerative medicine. ${ }^{9,10,17}$ Previous studies have
Correspondence: Carlos E Ambrosio Department of Basic Science, Faculty of Animal Science and Food Engineering, University of São Paulo, Av Duque de Caxias Norte 225, Pirassununga, São

Paulo, Brazil, CEP I3635-900

Tel +55 II 35654 III2

Email ceambrosio@usp.br 
characterized amniotic membrane-derived cells from full-term human, ${ }^{13,18-20}$ horse,${ }^{14}$ cat,${ }^{8,21}$ bovine, ${ }^{22}$ ovine,,${ }^{23}$ rat,${ }^{24}$ and canine ${ }^{25,26}$ placenta.

The present study was aimed at isolating and characterizing progenitor mesenchymal cells from cat amniotic membranes for future applications in cell therapy (eg, autologous and allogeneic transplantation assays). We gave special attention to teratogenicity evaluation of this cell lineage after transplantation into immunodeficient mice since it is a key point in cell therapy.

Carnivores have been considered an attractive animal model for human disease trials. ${ }^{8,25-27}$ Besides the similarity of many genetic and chronic diseases with humans, carnivores can be handled easily, opening up possibilities for conducting experiments with larger numbers of animals with more confident results.

\section{Materials and methods} Isolation and culture of cat AMSCs

All experiments and procedures were performed in accordance with the ethical principles in animal research by COBEA (Colégio Brasileiro de Experimentação Animal), and the study was approved by the Faculty of Animal Science and Food Engineering ethical committee (2011.1.1658.74.6).

The AMSCs were isolated from four fetal membranes ( \pm 45 days) collected after routine ovariohysterectomy in cats under general anesthesia. The placentas were carefully washed with sterile phosphate-buffered saline (PBS) and transported in sterile standard collection containers. The amniotic membrane was mechanically separated from the allantoic sac, then washed repeatedly with sterile PBS at room temperature to remove blood and debris. The pieces of cat amniotic membrane were minced and subjected to enzymatic digestion $\left(0.1 \%\right.$ collagenase solution at $\left.38.5^{\circ} \mathrm{C}\right)$. The products of digestion were passed through a $40 \mu \mathrm{m}$ nylon filter (BD Falcon nylon cell strainer, BD Biosciences, San Jose, CA, USA, catalog number 352340). The cell solution was centrifuged at $303 \mathrm{~g}$ for 5 minutes at room temperature. The supernatant was discarded, and the cell pellet were resuspended in $1 \mathrm{~mL}$ of Dulbecco's modified Eagle's medium/ Ham's F12 solution (1:1, LGC Biotecnologia Ltda, Cotia, Brazil, catalog number 05) supplemented with $10 \%$ fetal bovine serum, 1\% nonessential amino acids, 1\% L-glutamine, and $1 \%$ penicillin and streptomycin. The isolated cells were seeded in cell culture dishes with Dulbecco's modified Eagle's medium/F12 culture medium supplemented as described above at $38.5^{\circ} \mathrm{C}$ and with $5 \% \mathrm{CO}_{2}$. The culture medium was replaced 24 hours after initial plating to remove the nonadherent cells. When plated cells reached $80 \%-90 \%$ confluence, the culture was recovered using trypsin $0.25 \%$ (Tryple Express, Invitrogen, Carlsbad, CA, USA) for proliferation, in vitro differentiation, and immunophenotyping assays.

\section{Cell quantification and doubling time analysis}

The isolated cat AMSCs were counted using a Neubauer chamber. The trypan blue dye method (1:1) was used to access cell viability. Cell viability was expressed as a percentage of unstained (living) cells.

To determine the cell doubling time, AMSCs at passage one were seeded in triplicate in tissue culture dishes at a density of $1.3 \times 10^{3}$ cells $/ \mathrm{cm}^{2}$. Every 2 days, the culture was harvested using trypsin $(0.25 \%)$, counted, and replated at the same density. Similar procedures were performed in the subsequent passages until passage 10 . The population doubling time was calculated in each passage using the formula $\mathrm{Ct} / \mathrm{Cd}$, where $\mathrm{Ct}$ represents culture time between passage $\mathrm{n}$ and passage $\mathrm{n}+1$, and $\mathrm{Cd}$ represents the cell doubling. $\mathrm{Cd}$ was calculated using the formula $\mathrm{Cd}=\ln (\mathrm{nf} / \mathrm{ni}) / \ln 2$, where $\mathrm{nf}$ represents harvested cells and ni represents seeded cells. ${ }^{14}$

\section{Cryopreservation}

The cat AMSCs were frozen at passage 0 to passage 1 in Dulbecco's modified Eagle's medium supplemented with $45 \%$ fetal bovine serum and $10 \%$ dimethyl sulfoxide. The cells were frozen over 24 hours at $-80^{\circ} \mathrm{C}$ using a Mr Frosty freezing protocol, and were then transferred and stored in liquid nitrogen for 6 months. After thawing, some cells were used for proliferation assays and immunohistochemical studies, and others for in vitro differentiation.

\section{Characterization by flow cytometry}

The cat AMSCs were tested for immunoreactivity against CD73, CD90, and CD34 (goat polyclonal antibody, sc-7045, Santa Cruz Biotechnology Inc., Santa Cruz, CA, USA), as well as CD45 and CD79 (mouse monoclonal antibody, Santa Cruz Biotechnology Inc., sc-101839).

The cat AMSCs were harvested at passage 3 by trypsinization and $3 \times 10^{4}$ cells $/ \mathrm{mL}$ were transferred to Eppendorf tubes, washed twice with PBS, and fixed with $4 \%$ formaldehyde solution for 15 minutes at room temperature. After washing with PBS, the cells were blocked with bovine serum albumin (BSA) $2 \%$ for 45 minutes, washed with $0.2 \%$ BSA, and labeled with primary antibodies in $0.2 \%$ BSA (1:300) overnight at $4{ }^{\circ} \mathrm{C}$. The controls were incubated with $0.2 \%$ 
BSA instead of primary antibodies. After that, the cells were washed in PBS and incubated with secondary conjugated antibodies (1:300; Alexa Fluor ${ }^{\circledR} 488$ rabbit anti-goat IgG $[\mathrm{H}+\mathrm{L}] * 2 \mathrm{mg} / \mathrm{mL}^{*}$ catalog number A11078. Invitrogen and Alexa Fluor ${ }^{\circledR} 488$ goat anti-mouse $\operatorname{IgG}[\mathrm{H}+\mathrm{L}] * 2 \mathrm{mg} / \mathrm{mL}^{*}$ catalog number A11001, Invitrogen) for a duration of 45 minutes at room temperature in the dark. Labeled cells were transferred to Falcon tubes and analyzed by flow cytometry.

\section{Immunocytochemical analysis}

The cat AMSCs were harvested at passage 3 by trypsinization and counted, and $3 \times 10^{4}$ cells were seeded in chamber slides until cell confluence reached at least $90 \%$. The culture was then fixed with $4 \%$ formaldehyde solution for 15 minutes at room temperature. To block nonspecific staining, the cells were incubated with $1 \%$ BSA for 30 minutes at room temperature. After that, the cells were labeled overnight with primary antibodies in $0.1 \% \mathrm{BSA}(1: 50)$ at $4^{\circ} \mathrm{C}$ and then incubated with secondary conjugated antibodies (1:100) for 45 minutes at room temperature in the dark. In order to identify the nuclei, the slides were assembled with Hoescht 33342 stain solution $(10 \mu \mathrm{g} / \mathrm{mL})$ for 15 minutes at room temperature and examined by immunofluorescence microscopy. In negative controls, the primary antibodies were replaced with buffer solution (BSA 1\%) alone.

\section{In vitro differentiation studies}

Cells were seeded at passage 3 for all differentiation studies and cultured for 3 weeks at $38.5^{\circ} \mathrm{C}$ and in $5 \% \mathrm{CO}_{2}$. The controls were performed without induction medium.

For osteogenic differentiation, $4.2 \times 10^{3}$ cells $/ \mathrm{cm}^{2}$ were plated in osteogenic/adipogenic base medium (StemXVivo ${ }^{\mathrm{TM}}$, R\&D Systems Inc., Minneapolis, MN, USA, catalog number CCM007) supplemented with penicillin-streptomycin (100:1). After reaching 50\%-70\% confluence, the base medium was removed and replaced with complete osteogenic differentiation medium (StemXVivo osteogenic supplement and completed StemXVivo osteogenic/adipogenic base medium at a 1:20). The differentiation medium was subsequently changed every 4 days. After 21 days, the cells were harvested and analyzed by Alizarin red staining to assess the calcium deposits characteristic of osteogenic differentiation.

For adipogenic differentiation, $2.1 \times 10^{4} \mathrm{cells} / \mathrm{cm}^{2}$ were plated in StemXVivo osteogenic/adipogenic base medium supplemented with penicillin-streptomycin (100:1). The differentiation medium was subsequently changed every 4 days. After reaching 100\% confluence, the base medium were removed and replaced with complete adipogenic differentiation medium (StemXVivo adipogenic supplement and completed StemXVivo osteogenic/ adipogenic base medium at a 1:100). Fat vacuole formation was analyzed by Oil Red $\mathrm{O}$ staining 21 days after supplementation.

For chondrogenic differentiation, $1.5 \times 10^{5}$ cells $/ \mathrm{mL}$ were plated in Falcon tubes with $15 \mathrm{~mL}$ of chondrogenic base medium (R\&D Systems, catalog number CCM005) and penicillin-streptomycin (100:1). After 3 days, the tubes were centrifuged at $303 \mathrm{~g}$ for 5 minutes, the base medium was removed, and the cell pellet was resuspended with complete chondrogenic base medium supplemented with chondrogenic supplement medium (100:1; StemXVivo human/mouse chondrogenic supplement, R\&D Systems, catalog number CCM006). The differentiation medium was subsequently changed every 3 days. After 21 days, the tubes were centrifuged and the chondrogenic pellet was harvested and analyzed by toluidine blue staining.

\section{Teratoma formation assays}

The teratoma formation assays were performed at the Bioterium of Laboratory Animals (CEPTOX, FZEA, USP). Cat AMSCs (passage 4) were administered by intramuscular, subcutaneous, and intraperitoneal injection $\left(2.5 \times 10^{4}\right.$ cells/ injection) in three immunodeficient (BALB/c-Nu) mice. At 45 days post injection, the animals were euthanized and transplanted tissues were collected for histopathology assay. Thick tissue sections were stained with hematoxylin and eosin.

\section{Results}

\section{Isolation and culture of cat AMSCs}

After 48 hours in Dulbecco's modified Eagle's medium/F12 medium, the cat AMSCs demonstrated plate adherence and reached $70 \%-80 \%$ confluence. The cell morphology was similar to that of fibroblast-like cells and indistinguishable from bone marrow-derived MSCs (Figure 1A and B), and rapid cell clustering was observed (Figure 1C). The cells were extensively expanded up to nine passages without any morphologically visible changes. The cell doubling time increased with increasing passage number (Figure 1E), meaning that the proliferation rate was reduced with successive passages. Cell viability analysis revealed that the percentage of living cells in culture $(87.89 \%)$ was similar for both fresh and cryopreserved cells (Figure 1D) without depreciation of morphological or immunophenotypic features. 

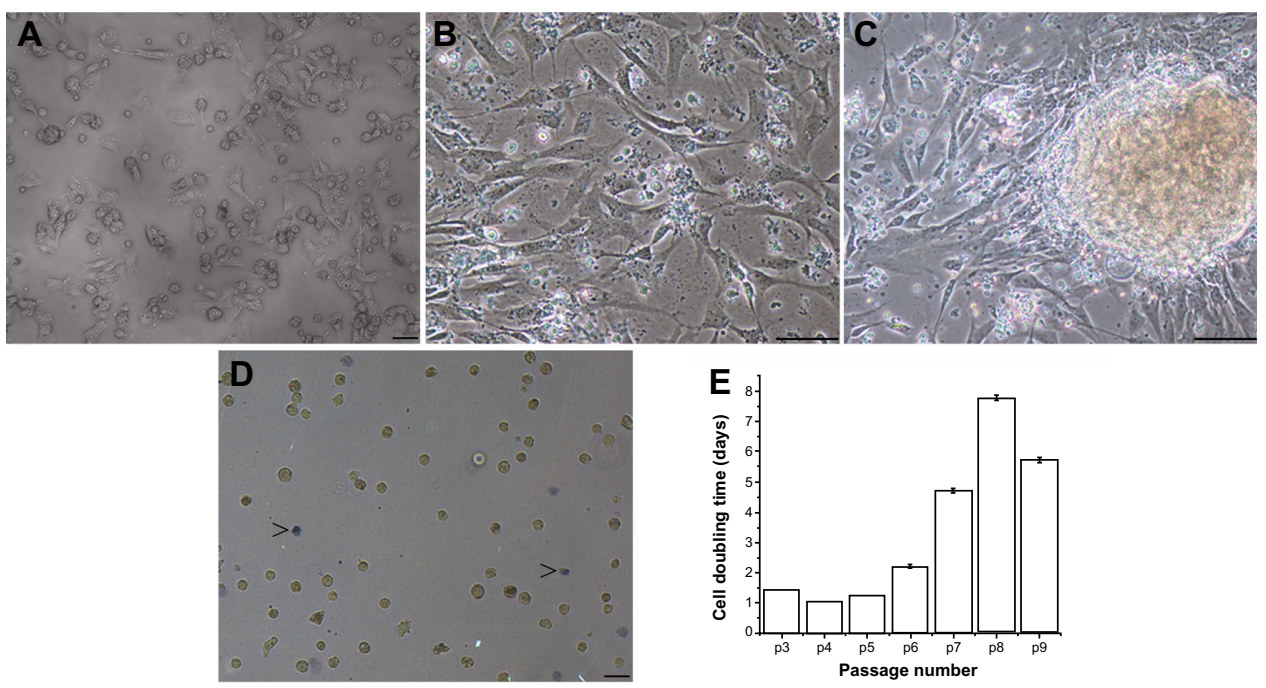

Figure I Feline amniotic membrane-derived cells. (A and B) Cell morphology, shows fibroblast-like cells adhering to plastic culture dishes (passage 0). (C) At high density, small cell clusters were observed. (D) Cell viability after cryopreservation; trypan blue staining (arrow heads). The percentages of dead cells were low in both fresh and cryopreserved cells (more dead cells stained in blue). (E) Cell doubling time for cat amnion-derived mesenchymal stem cells (expressed as the number of days required for cell number doubling) increased with increasing passage number. Bars: (A and D) $50 \mu \mathrm{m}$ (I0X); (B and C) $50 \mu \mathrm{m}(20 \times)$.

\section{Immunophenotyping assays}

Based on immunocytochemistry and flow cytometry, the cat AMSCs expressed the specific surface markers for MSCs, ie, CD73 (89.5\%) and CD90 (91.9\%), but the hematopoietic-specific markers CD34 (47\%), CD45 (1.7\%), and CD79 (29.1\%) were not expressed. The immunophenotypic profile was identical to that previously reported for bone marrow MSCs in other species (Figure 2A-L). However, 47\% of CD34 expression was detectable by flow cytometry, inconsistent with data from studies in humans (Figure 2L).

\section{In vitro differentiation}

When cultured with adipogenic differentiation medium, the cat AMSCs under goes adipogenic cells, as revealed by intracellular lipid droplet deposition 21 days after supplementation (Figure 3A and B). Cells induced towards osteogenic differentiation revealed calcium mineralization after Alizarin red staining (Figure 3D and E). Proteoglycans deposition in chondrogenic induced cells was revealed by toluidine blue staining 21 days after induction (Figure 3G).

\section{Teratoma formation}

When undifferentiated AMSCs were injected into immunodeficient (BALB/c-Nu) mice, no teratomas were observed by 4 weeks post injection, and the transplanted animals survived without difficulty (Figure 4A). Histopathological analysis of muscle, brain, lung, heart, liver, kidney, spleen, intestine, and lymph node fragments revealed no morphological changes
(Figures 4B and 5A-I). These results suggest that cat AMSCs are safer for use in cell therapy.

\section{Discussion}

Several studies have aimed to clarify the application of stem cells in regenerative medicine. Therefore, identification of alternative sources of stem cells, with optimal biological proprieties for cell therapy and easy yield or isolation, has become a crucial issue in current studies. The amniotic membrane is a promising source of alternative progenitor cells (AMSCs) with an enhanced ability to proliferate and differentiate into multiple lineages due to their primitive embryonic origin. In this study, we focused on this alternative source of MSCs, acquired through non-invasive methods and therefore ethically acceptable. The cat amnion is discarded after birth and could be collected after cesarean or ovariohysterectomy in pregnant cats.

We successfully isolated and expanded MSCs from cat amniotic membrane. These cells adhered to the culture dishes, showed fibroblastic-like morphology, and had high self-renewal capability. A sufficient level of homogeneity was reached at passage 3 . These data are consistent with previous studies in fetal membranes from $\operatorname{dog} s^{25}$ and recent trials involving feline fetal fluids and membranes. ${ }^{8}$ The population doubling time increased considerably from passage 7 . These values are higher than those reported in previous studies of horse $,{ }^{14} \mathrm{dog},{ }^{25}$ and feline fetal membranes. ${ }^{8,21}$ In the same way, in the data reported for dog, cat, and horse amnion-derived cells, the doubling time values tended to increase with increasing passages; however, 

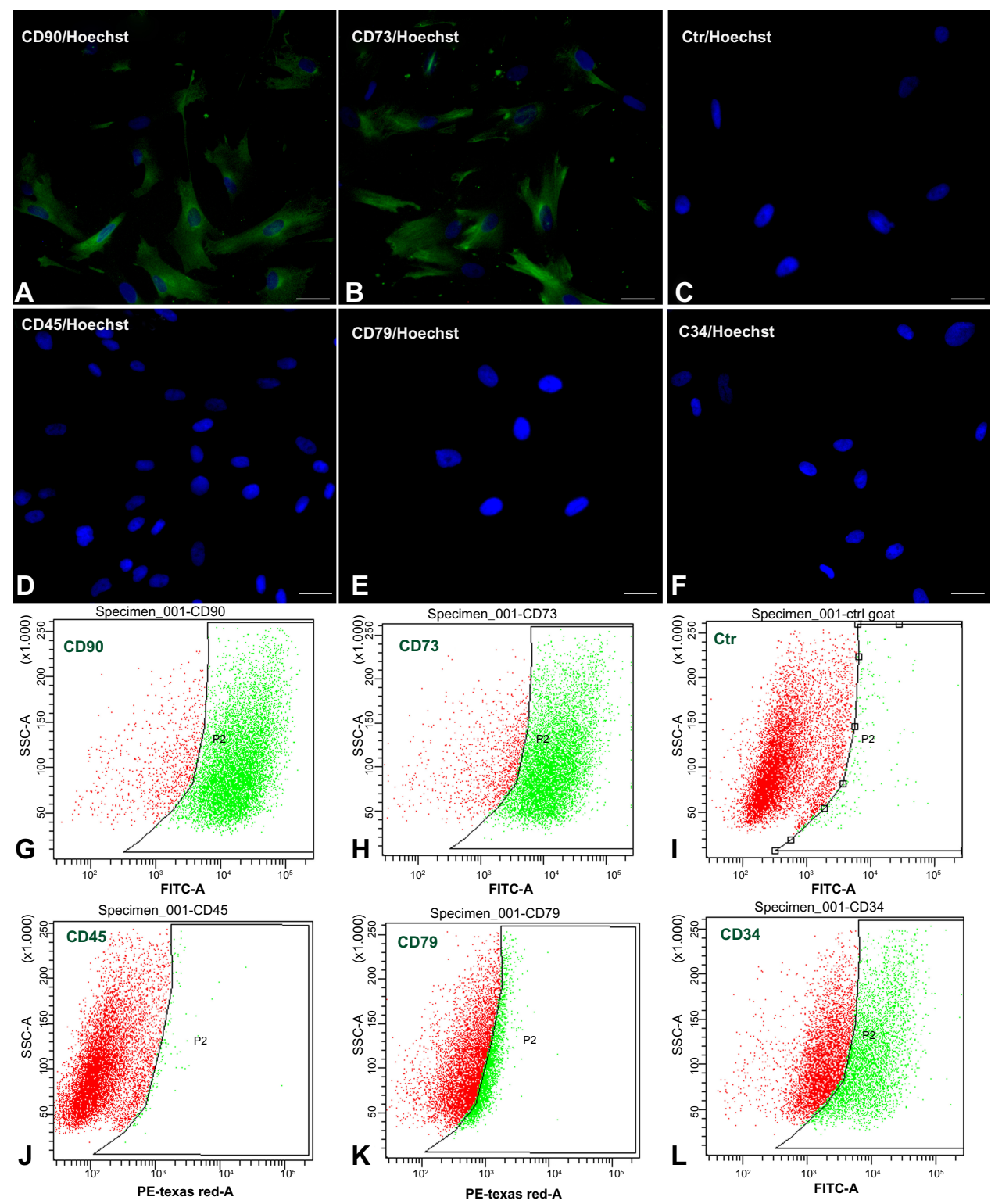

Figure 2 Photomicrographs and histogram overlays of immunophenotyping assays used to evaluate the expression patterns of specific surface markers. (A, B, $\mathbf{G}$ and $\mathbf{H})$ Mesenchymal stem cell markers (CD73 and CD90) were detected in cat-derived mesenchymal cells; however, hematopoietic markers (CD34, CD45, and CD79) were not detected (D-F and J-L). (C and I) Immunocytochemical and flow cytometry negative controls, respectively. The histograms represent the number of cells relative to the fluorescence intensity of the control (ctr) cells. (A-F) Bars $50 \mu \mathrm{m}(20 \times)$.

the values are not higher than 3 days. ${ }^{8,14,25}$ On the other hand, Rutigliano et $\mathrm{al}^{21}$ reported higher doubling times (5 days) in the first passage of culture.

Although population doubling time increased considerably with successive passages, e cell viability was high and similar for both fresh and cryopreserved cells. These data are in agreement with those reported for MSCs derived from the $\operatorname{dog}^{25}$ and suggest that these cell lineages are ideal for cell banking for future application in cell transplantation. On the other hand, amniotic epithelial cells (polygonal or columnarlike cells, derived from the amniotic epithelium $)^{21}$ were not observed in our primary cultures, confirming successful isolation of the MSC lineage from cat amnion. Further, when the cat AMSCs were cultured in high density at passage 0 , formation of small cell clusters was observed, suggesting strong cell-cell contact and signaling. Cell-cell contact and autocrine and paracrine signaling play a key role in the commitment and specification of MSC. ${ }^{2}$

The cat AMSCs expressed specific MSC antigen markers, CD73 and CD90, but not hematopoietic surface markers CD34, CD45, and CD79. This expression pattern was expected, and similar results were reported in previous studies of feline bone marrow-derived stem cells. ${ }^{28}$ These data confirm that amniotic membrane MSCs were successfully 

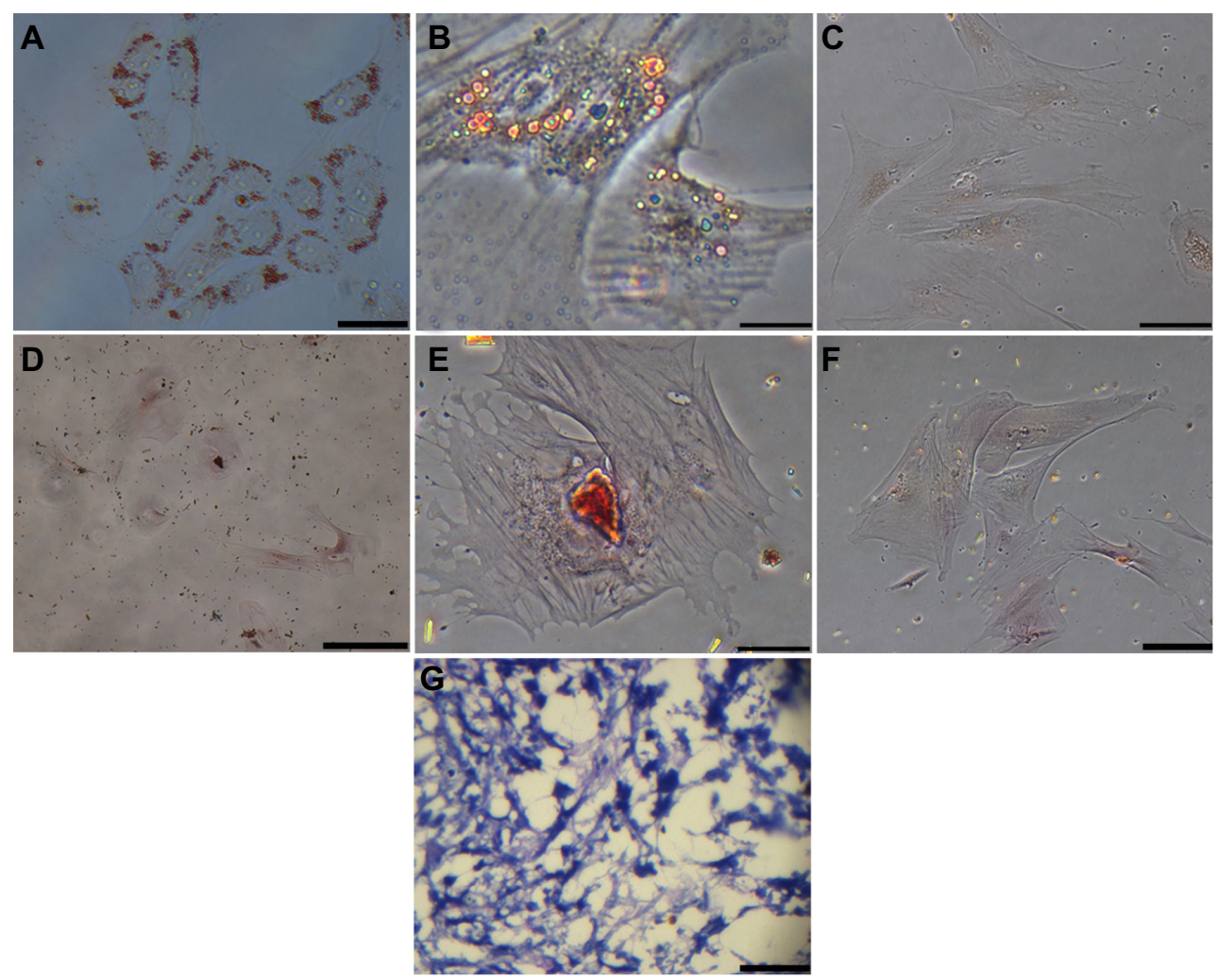

Figure 3 In vitro differentiation of the cat amnion-derived mesenchymal stem cells. (A and B) Oil Red O staining after adipogenic induction revealed lipid drops 2 I days after supplementation. (D and E) Alizarin red staining revealed mineralized matrix deposits after osteogenic induction. (G) Proteoglycans deposition in chondrogenic induced cells was reveled by blue toluidine staining 21 days after induction. (C and F) Oil Red $O$ and Alizarin red staining negative controls. (A and $\mathbf{G})$ Bars $20 \mu \mathrm{m}(20 \times)$. (B, C, E and $\mathbf{F}$ ) $10 \mu \mathrm{m}(40 \times)$. (D) $50 \mu \mathrm{m}(10 \times)$.

isolated without blood or debris contamination. However, this study demonstrated a high level of expression of CD34 at passage 4 , inconsistent with data from studies in humans. The explanation for this finding is that the AMSCs may have angiogenic potential, also suggested by other authors. ${ }^{8,14,29} \mathrm{In}$ both cell populations (AMSCs and amniotic epithelial cells) in the horse,$^{14}$ the CD34 marker started to be expressed at passage 5. This variation in membrane expression markers may occur from one passage to another because of acquisition
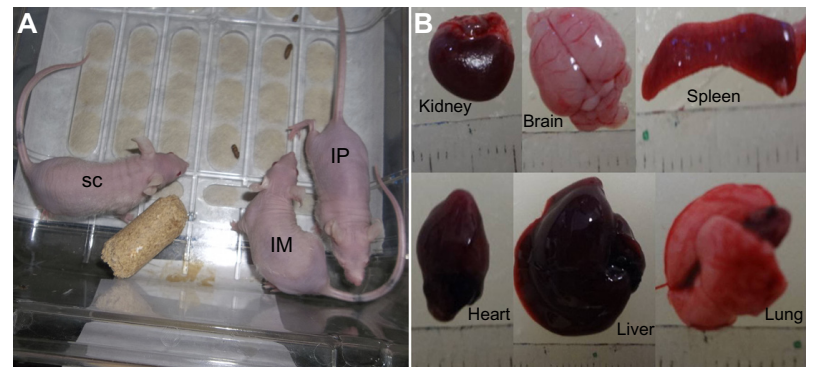

Figure 4 Amnion-derived mesenchymal stem cells were introduced into immunodeficient (BALB/c-Nu) mice via intramuscular, subcutaneous, and intraperitoneal injection $\left(2.5 \times 10^{4}\right.$ cells/injection). After four weeks post-injection, no teratomas were formed in the injected animals $(\mathbf{A})$ and the macroscopic analysis of the viscera in the all injected animals revealed no morphological changes (B).

Abbreviations: SC, subcutaneous; IM, intramuscular; IP, intraperitoneal. of angiogenic potential. On the other hand, our data could be related to the use of non feline-specific antibodies. Further investigations must be carried out in order to clarify these variations in expression of molecular markers in various stages of cell specification and/or use more specific techniques. Previous studies suggest that these cells are still of fetal origin and retain the properties of primitive multipotent stem cells. ${ }^{1,29,30}$

Recently, the AMSCs have awakened a great deal of interest in cell therapy due to their immunomodulatory properties, ease of accessibility, and high yield. Moreover, they have been shown to have a high ability to differentiate into different cell lineages. Using specific culture medium, we demonstrated that cat AMSCs differentiated into osteogenic, chondrogenic, and adipogenic cells. These results suggest that cat AMSCs may represent an important source of multipotent MSCs with properties identical to those of bone marrow-derived MSCs. The ability of MSCs to acquire a specific phenotype under defined conditions (microenvironment and interaction with soluble growth factor) is the critical issue for use of stem cells in regenerative medicine. Previous studies report the similarly high plasticity of MSCs derived from different organs post natally. $12,18,21,25,31$ 


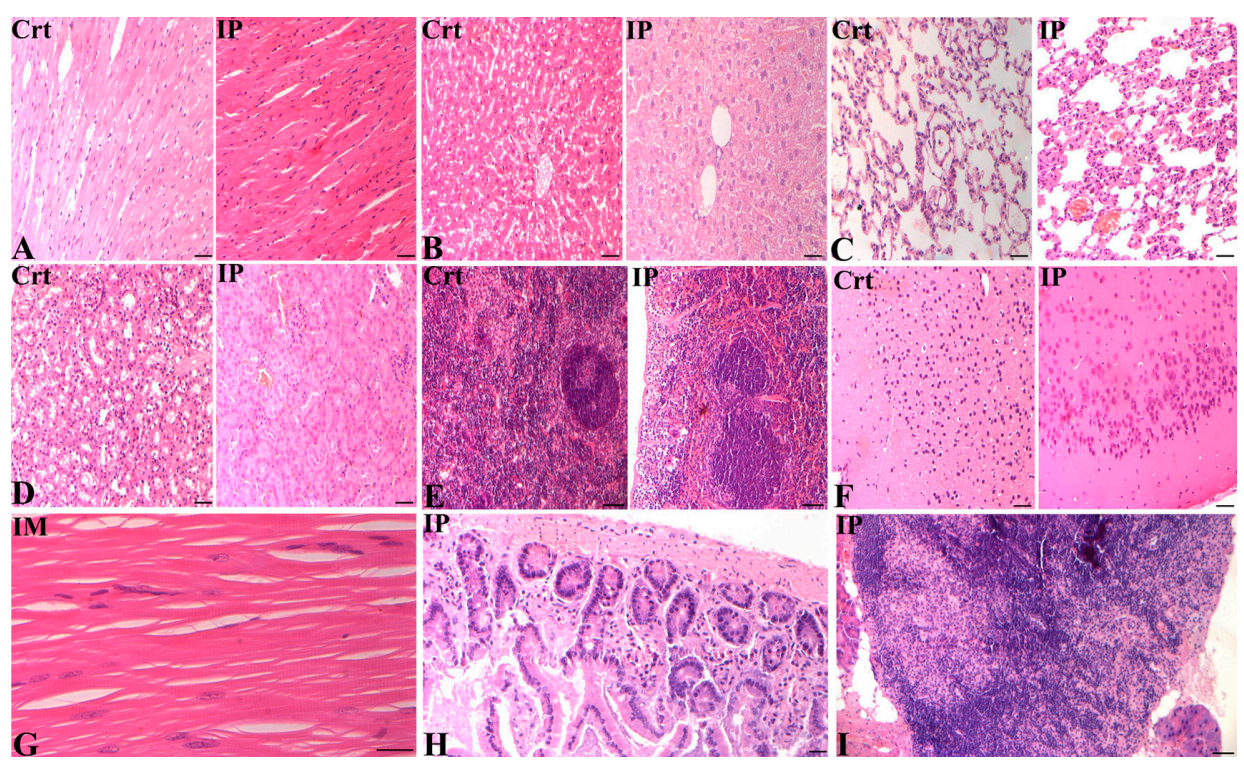

Figure 5 Photomicrographs of different organs collected from euthanized nude mice, four weeks post amnion-derived mesenchymal stem cell injection via the intramuscular (IM) and intraperitoneal (IP) routes. (A) Longitudinal section of cardiac muscle showing a common arrangement of muscle fibers with centralized nuclei. (B) Photomicrograph of the liver, central vein and numerous hepatic cords lining the sinusoidal capillaries. (C) Lung morphology, pulmonary alveoli, and alveolar ducts. (D) Cross-section of the kidney and overview of the glomeruli and renal tubules. (E) Cross-section of the spleen, normal distribution of lymphatic nodules, trabeculae, and white and red pulp. (F) Brain photomicrograph showing different layers of the cerebral cortex. (G) Longitudinal section of thigh skeletal muscle, nuclei in periphery of muscle fibers, and numerous transverse striations. (H) Small intestine, numerous glands immersed in the submucosal tissue. (I) Normal distribution of medullary cords and medullary sinuses of lymph node, with adipocytes in the periphery. (A, B, C, E and G) Bars $20 \mu \mathrm{m}$ (40×), (D, F and H) $20 \mu \mathrm{m}(20 \times)$, and (I) $50 \mu \mathrm{m}(20 \times)$.

Abbreviation: Ctr, control.

When cat AMSCs were injected into immunodeficient (BALB/c-Nu) mice, no tumors were generated by 4 weeks post injection, and the transplanted animals survived without difficulty. Photomicrographs of a number of organs collected from euthanized mice revealed no morphological changes when compared with the control group. This finding suggests that these cells can be used for cell transplantation without any risk of teratoma generation. Carnivores have been considered an attractive animal research model to study human disease. The findings in the feline model can be substantiated for future application in models of human disease, which are common between human and carnivores.

\section{Conclusion}

Based on these results, we conclude that the cat amniotic membrane may be a valuable alternative MSC source, and could be of interest to cell therapy researchers in a canine model system. One advantage of cat AMSCs is their nonteratogenicity, and they are safe for use in cell therapy.

\section{Acknowledgments}

We thank the Conselho Nacional de Pesquisa Brasil and the Ministério da Ciência e Tecnologia Moçambique for financial support.

\section{Disclosure}

The authors report no conflicts of interest in this work.

\section{References}

1. Ilancheran S, Michalska A, Peh G, Wallace EM, Pera M, Manuelpillai U. Stem cells derived from human fetal membranes display multilineage differentiation potential. Biol Reprod. 2007;77(3): 577-588.

2. Vidane AS, Zomer HD, Oliveira BM, et al. Reproductive stem cell differentiation: extracellular matrix, tissue microenvironment, and growth factors direct the mesenchymal stem cell lineage commitment. Reprod Sci. 2013;20(10):1137-1143.

3. Meirelles LS, Chagastelles PC, Nardi NB. Mesenchymal stem cells reside in virtually all post-natal organs and tissues. $J$ Cell Sci. 2006;119(11):2204-2213.

4. Mohr S, Portmann-Lanz CB, Schoeberlein A, Sager R, Surbek DV. Generation of an osteogenic graft from human placenta and placenta-derived mesenchymal stem cells. Reprod Sci. 2010;17(11): 1006-1015.

5. Brolio MP, Vidane AS, Zomer HD, et al. Morphological characterization of the progenitor blood cells in canine and feline umbilical cord. Microsc Res Tech. 2012;75(6):766-770.

6. Portmann-Lanz CB, Schoeberlein A, Huber A, et al. Placental mesenchymal stem cells as potential autologous graft for pre and perinatal neuroregeneration. Am J Obstet Gynecol. 2006;194(3): 664-673.

7. Fernandes RA, Wenceslau CV, Reginato AL, Kerkis I, Miglino MA Derivation and characterization of progenitor stem cells from canine allantois and amniotic fluids at the third trimester of gestation. Placenta. 2012;33(8):640-644.

8. Iacono E, Cunto M, Zambelli D, Ricci F, Tazzari PL, Merlo B. Could fetal fluid and membranes be an alternative source for mesenchymal stem cells (MSCs) in the feline species? A preliminary study. Vet Res Commun. 2012;36(2):107-118.

9. Lage-Consiglio A, Rossi D, Tassan S, Perego R, Cremonesi F, Parolini O. Conditioned medium from horse amniotic membrane-derived multipotent progenitor cells: immunomodulatory activity in vitro and first clinical application in tendon and ligament injuries in vivo. Stem Cells Dev. 2013;22(22):3015-3024. 
10. Insausti CL, Blanquer M, García-Hernández AM, Castellanos G, Moraleda JM.Amniotic membrane-derived stem cells: immunomodulatory properties and potential clinical application. Stem Cells Cloning. 2014;7: 53-63.

11. Huo S, Shi P, Pang X. Culture and identification of human amniotic mesenchymal stem cells. Chin Med Sci J. 2010;25(4):211-214.

12. Chen J, Lu Z, Cheng D, Peng S, Wang H. Isolation and characterization of porcine amniotic fluid-derived multipotent stem cells. PLos One. 2011;6(5):e19964.

13. Diaz-Prado S, Muinos-Lopez E, Hermida-Gomez T, et al. Isolation and characterization of mesenchymal stem cells from human amniotic membrane. Tissue Eng Part C. 2011;17(1):49-59.

14. Lage-Consiglio A, Corradetti B, Bizzaro D, et al. Characterization and potential applications of progenitor-like cells isolated from horse amniotic membrane. J Tissue Eng Regen Med. 2012;6(8):622-635.

15. Insausti CL, Alcaraz A, Garcia-Vizcaino EM, et al. Amniotic membrane induces epithelialization in massive posttraumatic wounds. Wound Repair Regen. 2010;18(4):368-377.

16. Plummer CE, Ollivier F, Kallberg M, et al. The use of amniotic membrane transplantation for ocular surface reconstruction: a review and series of 58 equine clinical cases (2002-2008). Vet Ophthalmol. 2009;12 Suppl 1:17-24.

17. Abdulrazzak H, Moschidou D, Jones G, Guillot PV. Biological characteristics of stem cells from foetal, cord blood and extraembryonic tissues. J R Soc Interface. 2010;7 Suppl 6:S689-S706.

18. Soncini M, Vertua E, Gibelli L, et al. Isolation and characterization of mesenchymal cells from human fetal membranes. J Tissue Eng Regen Med. 2007;1(4):296-305.

19. Mihu CM, Ciuca DR, Soritau O, Susman S, Mihu D. Isolation and characterization of mesenchymal stem cells from the amniotic membrane. Rom J Morphol Embryol. 2009;50(1):73-77.

20. Diaz-Prado S, Muinos-Lopez E, Hermida-Gomez T, et al. Multilineage differentiation potential of cells isolated from the human amniotic membrane. J Cell Biochem. 2010;111(4):846-857.

21. Rutigliano L, Corradetti B, Valentini L, et al. Molecular characterization and in vitro differentiation of feline progenitor-like amniotic epithelial cells. Stem Cell Res Ther. 2013;4(5):133.
22. Corradetti B, Meucci A, Bizzaro D, Cremonesi F, Lange-Consiglio A. Mesenchymal stem cells from amnion and amniotic fluid in the bovine. Reproduction. 2013;145(4):391-400.

23. Mauro A, Turriani M, Ioannoni A, et al. Isolation, characterization, and in vitro differentiation of ovine amniotic stem cells. Vet Res Commun. 2010;34:S25-S28.

24. Marcus AJ, Coyne TM, Rauch J, Woodbury D, Black IB. Isolation, characterization, and differentiation of stem cells derived from the rat amniotic membrane. Differentiation. 2008;76(2):130-144.

25. Uranio MF, Valentini L, Lange-Consiglio A, et al. Isolation, proliferation, cytogenetic, and molecular characterization and in vitro differentiation potency of canine stem cells from foetal adnexa: a comparative study of amniotic fluid, amnion, and umbilical cord matrix. Mol Reprod Dev. 2011;78(5):361-373.

26. Valentini L, Uranio MF, Consiglio AL, et al. Isolation, proliferation, and characterization of mesenchymal stem cells from amniotic fluid, amnion, and umbilical cord matrix in the dog. Reprod Fertil Dev. 2011;23(1):252-253.

27. Seo MS, Jeong YH, Park JR, et al. Isolation and characterization of canine umbilical cord blood-derived mesenchymal stem cells. JVet Sci. 2009;10(3):181-187.

28. Martin DR, Cox NR, Hathcock TL, Niemeyer GP, Baker HJ. Isolation and characterization of multipotential mesenchymal stem cells from feline bone marrow. Exp Hematol. 2002;30(8):879-886.

29. Alviano F, Fossati V, Marchionni C, et al. Term amniotic membrane is a high throughput source for multipotent mesenchymal stem cells with the ability to differentiate into endothelial cells in vitro. BMC Dev Biol. 2007;7:11.

30. Manuelpillai U, Moodley Y, Borlongan CV, Parolini O. Amniotic membrane and amniotic cells: Potential therapeutic tools to combat tissue inflammation and fibrosis? Placenta. 2011;32:S320-S325.

31. Chang Y-J, Hwang S-M, Tseng C-P, et al. Isolation of mesenchymal stem cells with neurogenic potential from the mesoderm of the amniotic membrane. Cells Tissues Organs. 2010;192(2):93-105.
Stem Cells and Cloning: Advances and Applications

\section{Publish your work in this journal}

Stem Cells and Cloning: Advances and Applications is an international, peer-reviewed, open access journal. Areas of interest in stem cell research include: Embryonic stem cells; Adult stem cells; Blastocysts; Cordblood stem cells; Stem cell transformation and culture; Therapeutic cloning; Umbilical cord blood and bone marrow cells; Laboratory,

\section{Dovepress}

animal and human therapeutic studies; Philosophical and ethical issues related to stem cell research. This journal is indexed on CAS. The manuscript management system is completely online and includes a quick and fair peer-review system. Visit http://www.dovepress.com/ testimonials.php to read real quotes from published authors. 